\title{
Colonoscopia: quando a preparação se torna uma complicação
}

Hugo Gaspar, ${ }^{1}$ Vânia Morais ${ }^{1}$

\section{RESUMO}

Introdução: A colonoscopia é um dos exames preconizados para o diagnóstico precoce do cancro do cólon e reto, estando este associado a incidência e a mortalidade elevadas no nosso país. Representa ainda a primeira linha de investigação perante a presença de sinais ou sintomas sugestivos de patologia do cólon ou do reto. Como qualquer procedimento médico, a colonoscopia não é isenta de complicações, que podem acontecer em diversas etapas: na preparação intestinal, na sedação/analgesia ou durante o exame colonoscópico propriamente dito. Este caso clínico ilustra uma complicação durante a preparação intestinal para a colonoscopia com picossulfato de sódio/citrato de magnésio, evidenciando o papel essencial do médico de família na gestão da saúde do doente.

Descrição do caso: Mulher de 56 anos, autónoma, com antecedentes pessoais de hipertensão arterial e doença hemorroidária, recorreu à consulta por episódios esporádicos de retorragia, acompanhados de dor anal e desconforto nos quadrantes abdominais inferiores, com alguns anos de evolução. Foi pedida colonoscopia para esclarecimento das queixas. Durante a preparação intestinal, a doente sofreu queda da própria altura, da qual resultou fratura parietal direita, com perda de conhecimento, motivando internamento hospitalar. A recuperação foi mais lenta do que o expectável e, decorridos dois anos, a doente mantém alterações da memória, nomeação e concentração, confirmadas em avaliação neuropsicológica. Fez várias tentativas de retomar a sua atividade profissional, mantendo ainda, no entanto, incapacidade para exercê-la.

Comentários: Embora as complicações graves da colonoscopia sejam incomuns, as suas consequências podem afetar a qualidade de vida dos doentes a curto, médio e longo prazo, devendo, por isso, ser ponderadas previamente à prescrição deste exame. Embora haja necessidade de gerir reduzidos tempos de consulta, é importante estar alerta para as possíveis complicações associadas às diversas etapas do exame, de forma a informar, esclarecer e capacitar os doentes sobre as suas consequências.

Palavras-chave: Colonoscopia; Catárticos; Efeitos adversos; Desequilíbrio hidroeletrolítico.

\section{INTRODUÇÃO}

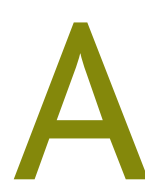

colonoscopia é o método de excelência para avaliação da mucosa do cólon e reto que, quando combinada com uma preparação intestinal adequada, permite diagnosticar patologia do cólon, reto e íleo terminal. Adicionalmente, apresenta uma vertente terapêutica que se traduz na excisão das lesões polipoides, ${ }^{1}$ pelo que se tornou um exame amplamente prescrito. ${ }^{2}$

A colonoscopia total representa a primeira linha de investigação perante a presença de sinais ou sintomas sugestivos de patologia do cólon ou reto, ${ }^{1}$ nomeada-

1. Médico Interno de Medicina Geral e Familiar. USF D. Jordão, ACeS Oeste Sul. mente retorragia ou pesquisa de sangue oculto nas fezes (PSOF) positiva, alterações do trânsito intestinal, dor abdominal dos quadrantes inferiores de causa não esclarecida, alterações do estado geral e vigilância de doentes submetidos previamente a polipectomias ou cirurgia colorretal por neoplasia. ${ }^{3}$

A colonoscopia é um procedimento invasivo, ${ }^{3}$ não sendo deste modo isento de complicações. ${ }^{1}$ Estas podem ocorrer nas diversas etapas que constituem o exame - preparação intestinal, sedação/analgesia ou exame colonoscópico propriamente dito. ${ }^{4}$ Embora $33 \%$ dos pacientes tenham reportado pelo menos um sintoma gastrointestinal transitório, as complicações graves associadas ao exame são raras. Numa revisão sistemática de 12 estudos conduzida em 2008 avaliou-se um 
total de 57.742 colonoscopias de rastreio, obtendo-se um rácio de 2,8 complicações graves por cada 1.000 procedimentos executados. Todavia, o risco de complicações será mais elevado se a colonoscopia for realizada com outro intuito que não o de rastreio. ${ }^{5}$ À semelhança de outros países da União Europeia, o rastreio oportunístico da população por colonoscopia total não está atualmente recomendado em Portugal. A evidência existente relativamente ao rastreio do cancro colorretal por colonoscopia total é limitada, sobretudo no que respeita ao hemicolon direito. ${ }^{6-8}$

As complicações referentes à preparação intestinal para a colonoscopia são atribuíveis tanto ao jejum como às soluções para limpeza do cólon, as quais podem induzir desconforto abdominal, tonturas, náuseas, vómitos e alterações hidroeletrolíticas. ${ }^{4} \mathrm{O}$ risco destas alterações parece estar relacionado com o tipo de preparação intestinal, idade e comorbilidades dos doentes. Existem no mercado diversas formulações de soluções laxantes destinadas à preparação intestinal. Idealmente devem ser fáceis de ingerir, com efeitos adversos mínimos a nível gastrointestinal e eletrolítico, de forma a assegurar uma maior segurança e adesão por parte do doente. Os fármacos mais frequentemente utilizados na preparação intestinal prévia à colonoscopia são o polietilenoglicol, o fosfato de sódio e o picossulfato de sódio/citrato de magnésio. ${ }^{9}$

A anestesia ou a sedação podem associar-se a outro tipo de complicações, como reações alérgicas medicamentosas, hipotensão, flebite no local da punção e raramente falência cardiorrespiratória. ${ }^{3}$

Em relação à colonoscopia per se, as complicações estão relacionadas com a técnica endoscópica e com os procedimentos terapêuticos, sendo a hemorragia e a perfuração intestinal as mais significativas. ${ }^{4}$

O caso clínico que se segue ilustra uma complicação consequente à preparação intestinal para a colonoscopia com uma solução contendo picossulfato de sódio/citrato de magnésio.

\section{DESCRIÇÃO DO CASO}

Identificação e história pessoal

Mulher de 56 anos de idade, leucodérmica, com antecedentes pessoais de hipertensão arterial, essencial sem evidência de lesões de órgão-alvo, dislipidemia, síndroma da coluna cervical (estreitamento C5-C6), perturbação do sono, doença hemorroidária e insuficiência venosa dos membros inferiores (submetida a safenectomia em 2016). Nega hábitos toxofílicos e alergias medicamentosas ou alimentares. Tem as vacinas preconizadas pelo Programa Nacional de Vacinação. Está medicada habitualmente com a associação de enalapril 20mg e hidroclorotiazida $12,5 \mathrm{mg}$, pravastatina $40 \mathrm{mg}$, trazodona $150 \mathrm{mg}$, dobesilato de cálcio $500 \mathrm{mg}$, estriol $1 \mathrm{mg} / \mathrm{g}$ e suplementos alimentares (Optimus@ e Neuralex().

\section{Caracterização sociofamiliar}

A doente tem seis anos de escolaridade, é empregada doméstica e integra uma família nuclear na fase VI do ciclo familiar de Duvall e de classe socioeconómica III (média), segundo a escala de Graffar. É casada há 34 anos e tem uma filha. Na Figura 1 apresenta-se o genograma desta família, bem como a representação da psicofigura de Mitchell. Desconhecem-se antecedentes familiares relevantes, excetuando os da mãe, que apresenta diabetes mellitus do tipo 2, hipertensão arterial complicada por hipertrofia ventricular esquerda, doença renal crónica, patologia osteoarticular degenerativa grave, anemia ferropénica e doença diverticular sigmoideia com episódios de melenas e retorragias associados.

\section{História da doença atual \\ Consulta programada (19/11/2015)}

A doente veio à consulta por queixas recorrentes de dor anal associada a desconforto nos quadrantes abdominais inferiores, com alguns anos de evolução, mas com agravamento nos últimos meses. Referia ainda episódios esporádicos de retorragias, contudo, sem alterações do trânsito gastrointestinal. Ao exame retal destacava-se um pequeno nódulo perianal mole, indolor, não justificativo das queixas. Realizara recentemente PSOF, cujo resultado se revelara positivo fraco, assim como hemograma, ionograma, creatinina sérica e eletrocardiograma, os quais não apresentaram alterações relevantes. Pediu-se, então, colonoscopia para esclarecimento da dor e das perdas hemáticas.

\section{Preparação para a colonoscopia (23/02/2016)}

$\mathrm{Na}$ véspera da colonoscopia, a doente iniciou a preparação intestinal com solução laxante de venda livre 


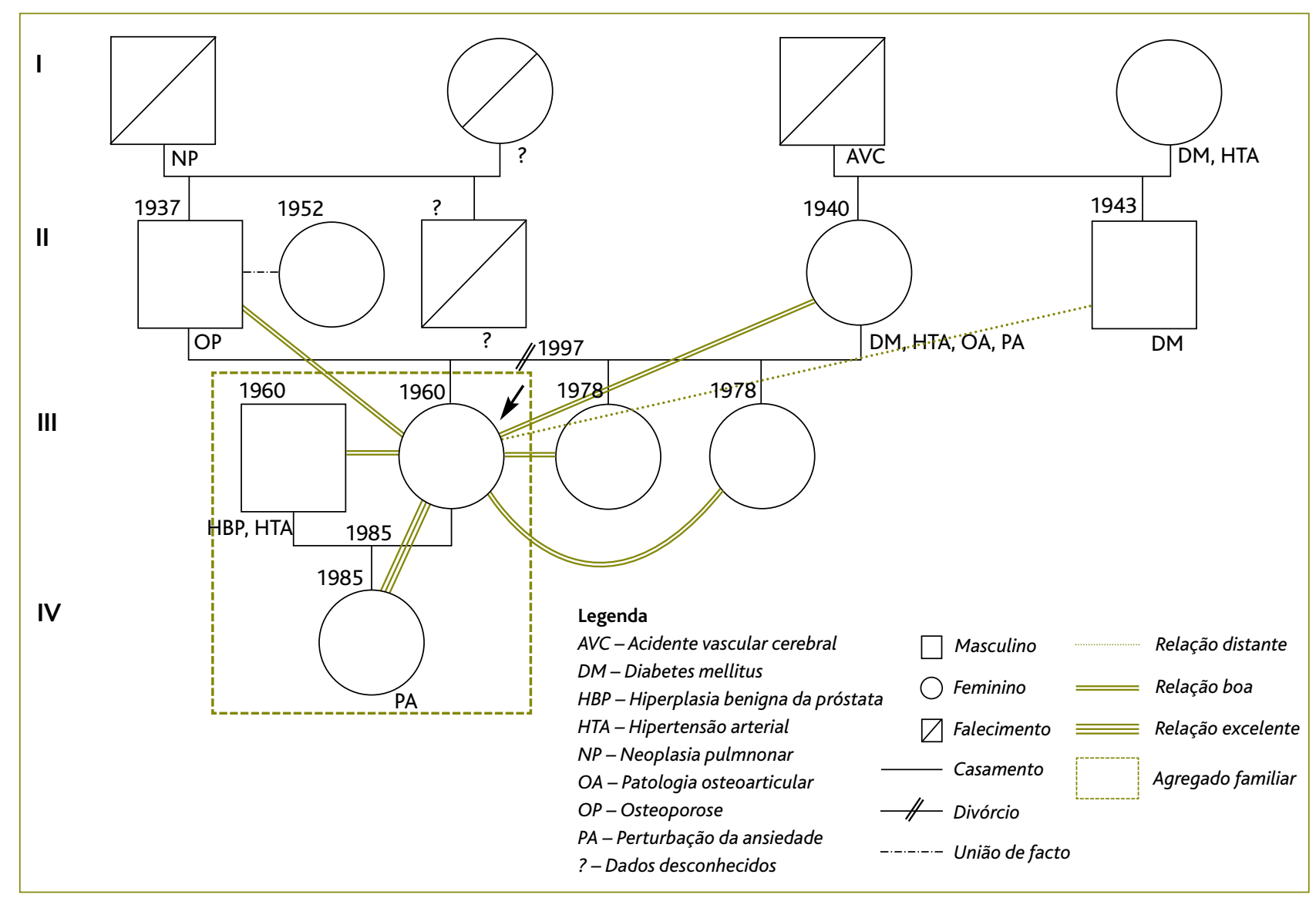

Figura 1. Genograma familiar (dados recolhidos em consulta, em março de 2018, durante entrevista à doente).

contendo picossulfato de sódio/citrato de magnésio (CitraFleet ${ }^{\circledR}$ ). Pela hora do jantar teve um vómito aquoso, acompanhado de sensação de fraqueza generalizada e arrepios, sem outros sintomas associados. Nessa mesma noite foi encontrada inconsciente pelos familiares, após queda da própria altura com perda de conhecimento.

\section{Serviço de urgência do hospital de referência (24/02/2016)}

À chegada ao serviço de urgência do hospital de referência apresentava-se em estado comatoso (Glasgow Coma Scale [GCS] 6), com necessidade de sedação, entubação orotraqueal e ventilação mecânica. Após a realização de tomografia computorizada crânio-encefálica (TCCE) constatou-se fratura parietal direita, pelo que foi transferida para o serviço de urgência do hospital central para observação por neurocirurgia.
Serviço de urgência do hospital central (24/02/2016)

Analiticamente destacava-se discreta leucocitose neutrofílica, depleção iónica iatrogénica grave (hiponatremia, hipocaliemia, hipocloremia e hipocalcemia) e elevação da creatinina quinase (CK igual a $5485 \mathrm{U} / \mathrm{L}$ ). Apresentava eletrocardiograma e radiografia torácica dentro da normalidade. Procedeu-se à correção hidroeletrolítica com melhoria gradual do estado geral (GCS 9-10), pelo que a doente foi extubada e transferida para o serviço de medicina interna.

Internamento no serviço de medicina interna (24/02 a 07/03/2016)

Decorridas $24 \mathrm{~h}$, a doente repetiu TCCE, que foi sobreponível à anterior, com ressonância magnética cerebral sem evidência de lesões endocraneanas de novo. Contudo, durante o internamento verificou-se um agravamento do estado de consciência (GCS 8), assim como 
episódios de mioclonias da hemiface esquerda e quadro febril sem foco infecioso aparente, pelo que realizou eletroencefalograma (EEG) e punção lombar, compatíveis com os diagnósticos de estado de mal epilético sem convulsão e meningite bacteriana sem isolamento de agente. Cumpridos 10 dias de antibioterapia e perante uma melhoria do estado geral, bem como dos parâmetros analíticos, a doente teve alta com o diagnóstico de coma multifatorial secundário a desequilíbrio hidroeletrolítico iatrogénico, meningite bacteriana e estado de mal epilético não convulsivo.

\section{Consulta programada (06/04/2016)}

Na primeira consulta na Unidade de Saúde Familiar posterior à alta hospitalar, a doente fez-se acompanhar da filha. Apresentava marcha hesitante com necessidade de recorrer ao apoio daquela, assim como discurso lentificado e lacónico, embora espontâneo e coerente. Referia humor irritável e lapsos de memória, quadro este que a incapacitava de realizar as suas atividades da vida diária.

Nos meses subsequentes foi retomando gradualmente o seu estado anterior, apesar de manter discretas alterações a nível do discurso, bem como queixas de perturbação da memória de curto prazo, concentração e vertigens ocasionais.

\section{Seguimento em consulta de medicina interna (desde o} internamento até à atualidade)

Após o internamento, a doente manteve seguimento em consulta de medicina interna, durante o qual foi manifestando diversos sintomas inespecíficos que motivaram referenciação pelo médico assistente hospitalar às consultas de otorrinolaringologia, neurologia e psiquiatria, determinando ainda internamento eletivo para estudo.

\section{Internamento eletivo no serviço de medicina interna} (29/03/2017)

A doente foi internada eletivamente para realização de preparação intestinal sob monitorização e posterior colonoscopia para exclusão de neoplasia e doença inflamatória intestinal, dado que mantinha hematoquézias persistentes, com aumento recente da frequência, acompanhadas de alternância do padrão de trânsito intestinal. A colonoscopia decorreu sem intercorrên- cias, não revelando lesões que justificassem as queixas, pelo que foram excluídas as hipóteses diagnósticas acima mencionadas. Face às alterações mnésicas mantidas durante o internamento, repetiu TCCE, que excluiu a existência de doença cerebrovascular isquémica ou hemorrágica. Realizou ainda EEG com cartografia que revelou atividade de base com ligeiro aumento dos ritmos rápidos, provavelmente devido a efeito medicamentoso, e atividade lenta focal temporal posterior à esquerda, escassa, sem atividade epilética. Suspendeu, assim, o levetiracetam que mantinha desde o internamento do ano anterior, sem se verificarem intercorrências neurológicas posteriores.

\section{Consulta externa de neurologia (18/08/2017)}

Dadas as queixas mnésicas, de nomeação e concentração foi pedida avaliação pela neurologia, que considerou tratar-se de perturbação de ansiedade com preocupação extrema da doente face ao seu estado de saúde, sendo referenciada para a consulta de psiquiatria.

Enquanto aguardava o agendamento da consulta de psiquiatria, por decisão própria, a doente realizou uma avaliação neuropsicológica que mostrou defeito generalizado na capacidade de atenção, defeito ligeiro na capacidade de iniciativa motora e grafomotora, na memória visual e na capacidade construtiva simples, assim como defeito moderado na capacidade de alternar entre dois estímulos visuais por lentificação. Revelou, ainda, sintomatologia ansiosa com tendência para a somatização.

A Figura 2 resume os principais acontecimentos da história da doença atual.

Decorridos dois anos e após várias tentativas de regresso ao trabalho, a doente mantém incapacidade para o exercício da sua atividade profissional, recorrendo mensalmente à Unidade de Saúde Familiar para renovação do certificado de incapacidade temporária, mantendo também seguimento em consulta de medicina interna.

\section{COMENTÁRIO}

A colonoscopia é um exame complementar de diagnóstico amplamente prescrito e de extrema importância como exame de primeira linha no esclarecimento de sinais ou sintomas sugestivos de patologia colorretal. No Quadro I encontram-se discriminadas as 


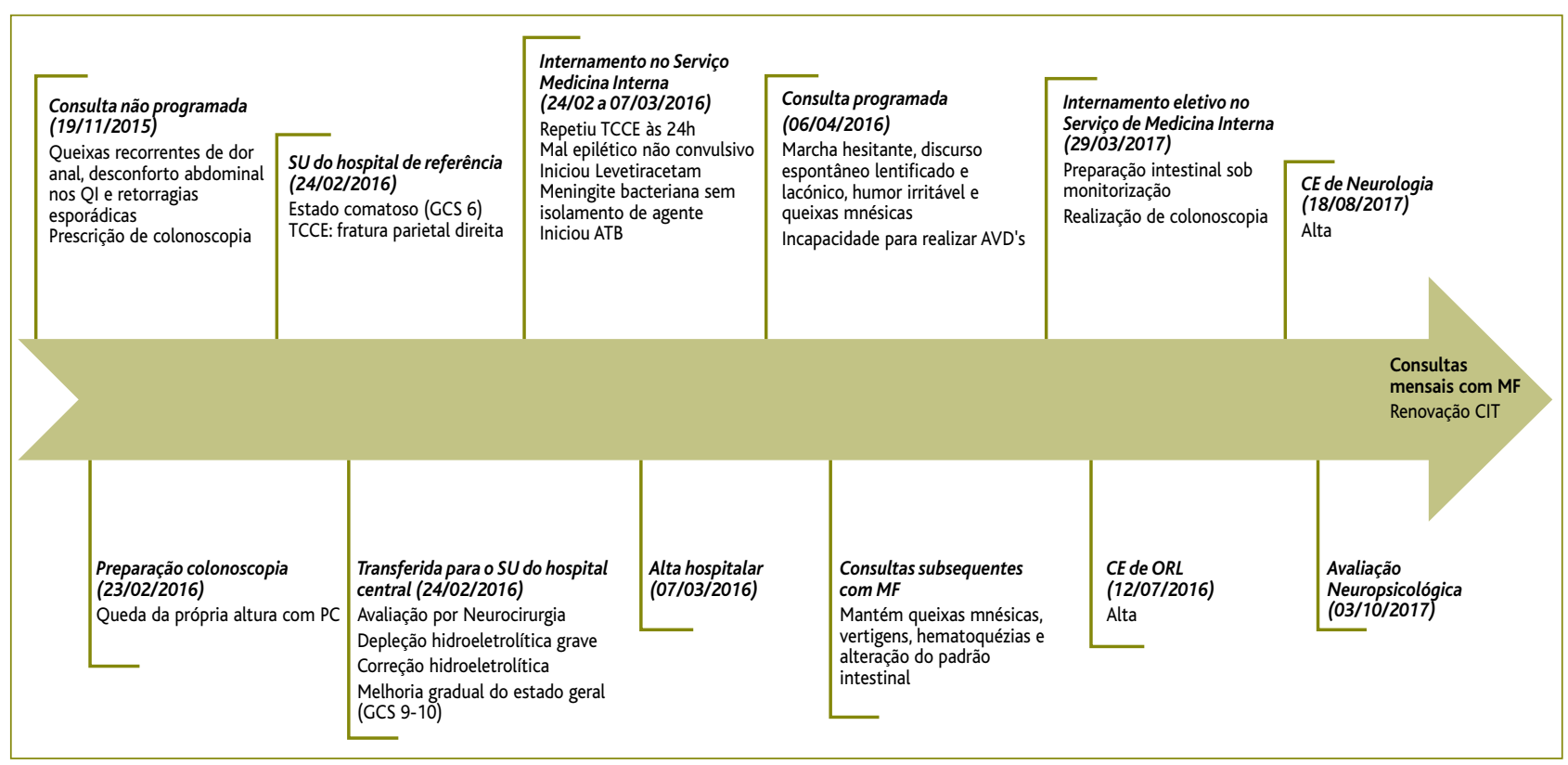

Figura 2. Resumo dos principais acontecimentos da história clínica.

Legenda: $\mathrm{QI}=$ quadrantes inferiores; $\mathrm{PC}=$ perda de conhecimento; $\mathrm{GCS}=$ Glasgow Coma Scale; $\mathrm{TCCE}=$ tomografia computorizada crânio-encefálica; $\mathrm{ATB}=$ antibioterapia; $\mathrm{AVD}$ = atividades de vida diárias; $\mathrm{CE}=$ consulta externa; $\mathrm{ORL}$ = otorrinolaringologia; $\mathrm{MF}$ = médico de família; $\mathrm{CIT}=$ certificado de incapacidade temporária.

indicações que devem motivar a realização de colonoscopia.

A otimização da colonoscopia correlaciona-se com a qualidade da preparação intestinal alcançada. Consideram-se excelentes condições de preparação quando estas permitem uma progressão do colonoscópio até ao nível pretendido, idealmente o cego, assegurando simultaneamente uma cuidada observação da mucosa colorretal. Adicionalmente, para se obter uma colonoscopia de qualidade o desconforto para o examinado deve ser mínimo e as complicações associadas à realização do procedimento inexistentes. ${ }^{1}$ Uma vez que se trata de um exame de observação direta, é imprescindível que o cólon se encontre livre de quaisquer resíduos fecais, ${ }^{3}$ pois poderão existir lesões planas ou de dimensões mais reduzidas que passam despercebidas na sua presença, particularmente as lesões sésseis do colón direito. ${ }^{10}$

Como referido na introdução, as complicações inerentes à preparação intestinal, nomeadamente as alterações hidroeletrolíticas, parecem relacionar-se, entre outras, com o tipo de solução laxante utilizado. ${ }^{4}$ Existem no mercado múltiplos agentes com diferentes formulações, sendo que os distúrbios eletrolíticos são mais frequentes naqueles à base de fosfato de sódio e picossulfato de sódio/citrato de magnésio, nos indivíduos com mais de 65 anos ou medicados com diuréticos tiazídicos, inibidores da enzima de conversão da angiotensina, beta-bloqueantes ou antidepressivos, ${ }^{6}$ bem como em doentes com compromisso renal, hepático, insuficiência cardíaca ${ }^{4}$ ou gastrectomizados. ${ }^{7}$ Perante estas situações devem ser preferidas soluções intestinais isosmóticas à base de macrogol (polietilenoglicol), ${ }^{9}$ uma vez que não alteram significativamente os parâmetros vitais ou o equilíbrio hidroeletrolítico. ${ }^{4}$

Este caso clínico ilustra uma complicação consequente à preparação intestinal prévia à colonoscopia, realizada em contexto de investigação perante um quadro de retorragia, dor abdominal persistente nos quadrantes inferiores e PSOF positiva. Apesar de a doente ter idade inferior a 65 anos e não apresentar qualquer uma das patologias descritas anteriormente, encontrava-se medicada com a associação de anti-hipertensores enalapril 20mg e hidroclorotiazida $12,5 \mathrm{mg}$ e com um antidepressivo atípico (trazodona). Adicionalmente, é ainda de considerar o facto de a doente nos dias prévios à toma da solução laxante ter diminuído o apor- 
QUADRO I. Indicações para a realização de colonoscopia' ${ }^{1}$

a) Pesquisa de sangue oculto nas fezes positiva no âmbito do rastreio oportunístico do cancro do cólon ou do reto;

b) História familiar de neoplasia do cólon e reto;

c) Hemorragia digestiva: retorragia/hematoquézia, presença de sangue oculto nas fezes, anemia ferropénica e melenas após realização de endoscopia digestiva alta que não tenha evidenciado lesões que a possam explicar;

d) Vigilância após resseção de neoplasia;

e) Dor abdominal persistente nos quadrantes inferiores e/ou alteração recente dos hábitos intestinais;

f) Diarreia crónica inexplicável;

g) No contexto da doença inflamatória intestinal;

h) Observação de todo o cólon, tendo em vista o diagnóstico de lesões neoplásicas síncronas em utentes com neoplasia ressecável do cólon ou do reto;

i) Realização de ato terapêutico, designadamente excisão de lesão, repermeabilização luminal ou hemostase;

j) Esclarecimento de imagens patológicas obtidas por outros métodos complementares de diagnóstico;

k) Identificação intraoperatória de lesão inaparente à cirurgia;

l) Marcação de uma neoplasia para posterior excisão cirúrgica;

m) Descompressão de megacólon agudo (não tóxico) ou de volvo da sigmoide;

n) Extração de corpo estranho;

o) No quadro da investigação de sintomas constitucionais não esclarecidos, devidamente fundamentado no processo clínico.

te calórico e aumentado a ingesta hídrica, não só com o intuito de proporcionar maior limpeza do lúmen intestinal, mas também consequente à ansiedade pela aproximação da data do exame. Assim, posteriormente à ingestão do agente picossulfato de sódio/citrato de magnésio, a doente iniciou um quadro de tonturas e náuseas, com episódio de vómito aquoso associado, bem como depleção iónica iatrogénica grave (hiponatremia, hipocaliemia, hipocloremia e hipocalcemia) e rabdomiólise subsequente.

A rabdomiólise poderá ter uma etiologia multifatorial; contudo, no contexto deste caso clínico, as lesões musculares traumáticas, os fármacos e as alterações eletrolíticas encontram-se categorizados entre as suas potenciais causas. ${ }^{12}$ Nestas últimas inclui-se a rabdomiólise induzida por hiponatremia, uma situação relativamente rara e cujo mecanismo fisiopatológico não se encontra ainda bem estabelecido. Alguns dos fatores que suscetibilizam à ocorrência desta síndroma são a polidipsia psicogénica, determinadas terapêuticas farmacológicas (nomeadamente diuréticos tiazídicos e inibidores de bomba de protões), distúrbios endocrinológicos e a iatrogenia (após cirurgia prostática ou preparação de colonoscopia com manitol). ${ }^{11}$ No contexto deste caso clínico, a rabdomiólise traduziu-se numa elevação da creatinina quinase, sem lesão renal aguda associada e com evolução favorável durante o internamento, sendo, no entanto, difícil estabelecer uma relação causal. Assume-se, contudo, que a rabdomiólise poderá ser consequente de uma hiponatremia secundária a perdas gastrointestinais resultantes da preparação intestinal, com provável agravamento face ao subsequente traumatismo sofrido pela doente.

A recuperação foi mais lenta do que o expectável e, decorridos dois anos, a doente mantém incapacidade para o exercício da sua atividade profissional. Concomitantemente, desde 2016 é notório um aumento substancial do número de consultas. Excluindo as consultas mensais na USF para renovação do certificado de incapacidade temporária, a doente recorreu à unidade em múltiplas ocasiões com sintomas vagos e inespecíficos - cefaleia incaracterística, mialgias, dor abdominal sem causa aparente, infeções agudas do trato respiratório superior. Estas queixas estão de acordo com o relatado na avaliação neuropsicológica - tendência para a somatização. 
Ao nível dos cuidados de saúde primários, a somatização representa uma realidade com que diariamente se contacta. A procura de uma causa orgânica leva a que se verifique um elevado consumo de recursos de saúde por parte destes doentes, traduzindo-se numa sobreutilização de consultas em diferentes especialidades com o intuito de procurar uma segunda opinião fundamentada por exames complementares de diagnóstico desnecessários, assim como, em última instância, numa polimedicação desmesurada. ${ }^{13-15}$ Contudo, nenhuma destas atitudes parece ter impacto na incapacidade funcional que impede o doente de exercer a sua atividade laboral. ${ }^{15} \mathrm{Assim}$, o médico de família, pela sua abordagem holística, assume o papel de gestor e integrador de cuidados, exercendo a advocacia do doente, de forma a capacitá-lo e ajudá-lo na tomada de decisões relativas à sua própria saúde. ${ }^{16}$

A família da doente tem desempenhado um papel significativo ao longo deste processo. A alteração da dinâmica familiar com aumento transitório da dependência da doente condicionou uma readaptação da família. O sentimento de entreajuda e coesão dos elementos da família, bem como o seu inconformismo face às queixas da doente, estimularam a procura incessante de respostas, apesar das opções terapêuticas limitadas.

Globalmente admite-se que a incidência real dos eventos adversos associados à colonoscopia seja subestimada em número e impacto, até porque uma proporção significativa de doentes sofre complicações $m i$ nor até alguns dias após o exame, ${ }^{17}$ as quais não são reportadas. Como tal, durante a redação do artigo e após revisão do processo, procedeu-se à notificação desta reação medicamentosa adversa ao Infarmed. Embora a colonoscopia seja um procedimento relativamente seguro, com baixo índice de complicações graves, ${ }^{4,17} \mathrm{es-}$ tas podem afetar consideravelmente a qualidade de vida dos doentes. A colonoscopia é um procedimento invasivo, com incómodo associado, pelo que se poderá impor a necessidade de prescrição de terapêutica sintomática, quando indicada, devendo ser monitorizadas as complicações, particularmente nos doentes de maior risco. ${ }^{18}$

Atualmente debate-se a prescrição excessiva de exames complementares de diagnóstico e os seus potenciais malefícios na saúde, tendo este caso clínico como principal objetivo consciencializar os médicos quanto aos potenciais riscos inerentes à sua realização. Numa altura em que são impostos indicadores de contratualização que avaliam a proporção de doentes submetidos a determinados rastreios oncológicos e em que os sistemas de registo clínico alertam para os rastreios em falta, deve estar sempre presente que qualquer exame prescrito, mesmo com intuito de rastreio oncológico, terá complicações inerentes que não deverão ser desconsideradas.

Embora seja exigida uma gestão rigorosa do tempo de consulta, frequentemente desajustado às necessidades do doente, cabe ao médico de família conhecer as possíveis complicações inerentes às diversas etapas de cada exame complementar de diagnóstico e transmiti-las aos doentes. O processo implica uma decisão partilhada, onde os riscos e benefícios do exame devem ser previamente discutidos com o doente, enfatizando o seu potencial diagnóstico e as suas implicações, sem, contudo, descurar as complicações que lhe estão atribuídas. Assim, o médico de família assume uma posição privilegiada, contribuindo para gerar mais e melhor conhecimento junto da população. Compete-lhe a função de informar, esclarecer e capacitar os doentes sobre as consequências de cada exame para que, perante a manifestação de alguma complicação, esta possa ser precocemente identificada com o intuito de providenciar, de forma célere, as medidas adequadas. Dever-se-á contrariar o impulso de descurar este passo crucial na prescrição de exames complementares de diagnóstico, pois «perder tempo» é «ganhar saúde».

\section{REFERÊNCIAS BIBLIOGRÁFICAS}

1. Direção-Geral da Saúde. Colonoscopia diagnóstica/terapêutica no adulto: norma n. ${ }^{\circ}$ 004/2014, de 31/03/2014, atualização em 12/09/2017. Lisboa: DGS; 2014.

2. Despacho n. ${ }^{\circ}$ 3756/2014, de 11 de março. Diário da República. $2^{\text {a Sé- }}$ rie(49):6600-1.

3. Rex DK, Petrini JL, Baron TH, Chak A, Cohen J, Deal SE, et al. Quality indicators for colonoscopy. Am J Gastroenterol. 2006;101(4):873-5.

4. Loureiro JF, Corrêa PA, Paccos JL, Rossini GF, Silva RM, Perecco TB, et al. Complicações em colonoscopia [Complications in colonoscopy]. GED Gastroenterol Endosc Dig. 2013:32(2):44-9. Portuguese

5. Fisher DA, Maple JT, Ben-Menachem T, Cash BD, Decker GA, Early DS, et al. Complications of colonoscopy. Gastrointest Endosc. 2011;74(4): 745-52.

6. Pignone M, Rich M, Teutsch SM, Berg AO, Lohr KN. Screening for colorectal cancer in adults at average risk: a summary of the evidence for 
the U.S. Preventive Services Task Force. Ann Intern Med. 2002;137(2): 132-41.

7. Costa AR, Silva S, Ferreira PM, Carmo I, Barros H, Lunet N. Rastreios oncológicos em Portugal. In: Ferreira PN, Lunet N, Silva S, editors. A informação sobre saúde dos Portugueses: fontes, conhecimentos e comportamentos. Lisboa: Associação para Investigação e Desenvolvimento da Faculdade de Medicina; 2017. p. 173-91.

8. Segnan N, Patnick J, von Karsa L, editors. European guidelines for quality assurance in colorectal cancer screening and diagnosis. Luxembourg: Publications Office of the European Union; 2010. ISBN 9789279164354

9. Costa JM, Soares JB. Symptomatic hyponatremia after bowel preparation: report of two cases and literature review. Acta Med Port. 2017;30 (11):824-6.

10. Von Karsa L, Patnick J, Segnan N, Atkin W, Halloran S, Lansdorp-Vogelaar I, et al. European guidelines for quality assurance in colorectal cancer screening and diagnosis: overview and introduction to the full supplement publication. Endoscopy. 2013;45(1):51-9.

11. Aguiar DT, Monteiro C, Coutinho P. Recurrent rhabdomyolysis secondary to hyponatremia in a patient with primary psychogenic polydipsia. Rev Bras Ter Intensiva. 2015;27(1):77-81.

12. Rosa NG, Silva G, TeixeiraA, Rodrigues F, Araújo JA. Rabdomiólise [Rhabdomyolysis]. Acta Med Port. 2005;18(4):271-81. Portuguese

13. Luo Z, Goddeeris J, Gardiner JC, Smith RC. Costs of an intervention for primary care patients with medically unexplained symptoms: a randomized controlled trial. Psychiatr Serv. 2007;58(8):1079-86.
14. Dowrick C, Gask L, Hughes JG, Charles-Jones H, Hogg JA, Peters S, et al. General practitioners' views on reattribution for patients with medically unexplained symptoms: a questionnaire and qualitative study. BMC Fam Pract. 2008;9:46.

15. Servan-Schreiber D, Kolb NR, Tabas G. Somatizing patients - Part I: practical diagnosis. Am Fam Physician. 2000;61(4):1073-8.

16. WONCA Europa. A definição europeia de medicina geral e familiar (Clínica geral/Medicina familiar). WONCA Europa; 2002.

17. De Jonge V, Sint Nicolaas J, Van Baalen O, Brouwer JT, Stolk MF, Tang TJ, et al. The incidence of 30-day adverse events after colonoscopy among outpatients in the Netherlands.Am J Gastroenterol. 2012;107(6):878-84.

18. Sherid M, Samo S, Sulaiman S. Complications of colonoscopy. In: Bustamante $\mathrm{M}$, editor. Colonoscopy and colorectal cancer screening: future directions. IntechOpen; 2013. p. 215-40.

\section{CONFLITO DE INTERESSES}

Os autores declaram não ter quaisquer conflitos de interesse.

\section{ENDEREÇO PARA CORRESPONDÊNCIA \\ Hugo Gaspar \\ E-mail: hugodgaspar@gmail.com \\ https://orcid.org/0000-0001-5901-7550}

Recebido em 09-08-2018

Aceite para publicação em 02-04-2019

\section{ABSTRACT}

\section{COLONOSCOPY:WHEN PREPARATION BECOMES A COMPLICATION}

Introduction: Colonoscopy is recommended for colorectal cancer early diagnostic due to its elevated incidence and mortality in Portugal. It is the first-line screening test when suggestive colon or rectal signs and symptoms are present. Like any other medical procedure, colonoscopy is not exempt from complications, which can happen in several stages: in the bowel preparation, in sedation/analgesia or during the colonoscopic examination itself. This is a case report of an intestinal preparation-related complication for colonoscopy with sodium picosulfate/magnesium citrate, proving the essential role of the family physician as a manager of the patient's health.

Case report: Fifty-six-year-old woman, autonomous regarding her daily activities, with a history of arterial hypertension and hemorrhoidal disease, presented to our medical center for sporadic rectal bleeding episodes, plus anal pain and lower quadrant discomfort with several years of evolution. A colonoscopy was requested for diagnostic investigation. During the intestinal preparation, the patient had a syncopal episode resulting in a right parietal fracture with conscience loss and requiring hospitalization. Recovery was longer than expected and, after two years, the patient remains with memory, naming and concentration deficits confirmed by neuropsychological evaluation. Several attempts for resuming professional activity were made; however, the patient was unable to do it.

Commentary: Although colonoscopy complications are uncommon, their outcome can affect the quality of patients' life in the short, medium or long term and, therefore, should be considered before prescribing the exam. Even though managing the reduced consultation time is a necessity, it is important to be alert to the possible complications stage-related with the procedure in order to inform, clarify and capacitate our patients about its consequences.

Keywords: Colonoscopy; Cathartics; Adverse effects; Electrolyte imbalance. 